Chapter 4 Unveiling early Earth photochemistry through experimental simulations of planetary atmospheres

N. Carrasco ${ }^{a}$ and T. Gautier ${ }^{a}$.

a Université Paris-Saclay, UVSQ, CNRS, LATMOS, 78280, Guyancourt, France

${ }^{*}$ corresponding email address: nathalie.carrasco@latmos.ipsl.fr

\title{
ABSTRACT/WEB SUMMARY:
}

This chapter summarizes the contributions of the early Earth atmosphere between late Hadean and early Archean eons $(4.3$ to $3.7 \mathrm{Ga})$ to sustain the emergence of life: influencing both the climate and prebiotic chemical synthesis through photochemistry. Almost no geological constraints remain on these early ages so that chemical scenarios are largely explored through experimental simulation. Main scientific results through time are given, from the pioneer experiment by Miller and Urey in the 50's, up to the most recent studies focusing on high altitude photochemistry. 


\subsection{Habitability of the Early Earth}

The oldest fossils discovered on Earth are stromatolites, sedimentary structures produced by cyanobacteria and dating from $3.7 \mathrm{Ga}^{1}$. These first archean forms of life dominated and spread on the early Earth during several $\mathrm{Ga}$, giving their name to the geological epoch ranging between 4 and $2.5 \mathrm{Ga}$ : the Archean eon. The previous and first eon of the Earth, Hadean, ranged between 4.5 and $4.0 \mathrm{Ga}$ (see Figure X.1). Geological evidences on the Hadean are very limited. However we know that liquid water, a criterion essential to the emergence of life, was already present at the surface since $4.3 \mathrm{Ga}$, as attested by the oxygen isotopic composition of zircons ${ }^{2}$. The appearance of life at the end of Hadean is therefore possible even if no clear geological record remains to support the occurrence of such an event ${ }^{3}$. In the following "early" and "late" Hadean will refer to stages respectively before and after the water oceans formation.

This leads us to question the habitability of the early Earth during the Hadean and the Archean eons. What were the environmental conditions at stake that ensured the emergence and development of life on the planet? To sustain life, the Earth should have hosted a water ocean, an energy source to promote biological processes, and a carbon-based polymeric chemistry providing building blocks for life ${ }^{4}$. A stable atmosphere over geological time spans is involved in all of these criteria. Atmospheric stability will in particular depend on a limited erosion by stellar winds, related to atmospheric composition the presence and strength of a magnetosphere and the stellar activity ${ }^{5}$. A dense atmosphere protects the planet surface from damaging UV radiations and enables to accumulate organic, and life to evolve. Atmospheric greenhouse effect also ensures stable climate conditions for maintaining liquid water on the surface. The atmosphere of the early Earth is therefore strongly related to its habitability.

The aim of this first section is to gather the information we have on the atmospheric environmental conditions that were at stake on the early Earth, ensuring the habitability of the planet when life appeared and expanded.

\section{1. 1. Origin of volatiles on the early Earth}

The solar system is partitioned in two chemically different regions: a dry inner solar system, which includes the four telluric planets and the inner main asteroids belt, and a water-rich outer solar system, with the four giant planets, their numerous icy satellites, primitive asteroids and transneptunian objects. This present dichotomy reveals a fossilized temperature ramp and a snow-line in the vicinity of the inner asteroid belt that controlled the volatile content in the early solar system ${ }^{6}$. The snow line actually indicates that the early Earth lost most of its volatiles and thus its hypothetical primary atmosphere during the first steps of the planet formation. If a 
residual trace of this primitive $\mathrm{H}_{2}$-rich atmosphere is not discarded, its further contribution to the Earth atmosphere became presumably minor. The volatiles, water and organic molecules, on the early Earth were therefore progressively brought by the permanent bombardment of asteroids and comets from the outer solar system that occurred massively during Hadean ${ }^{7,8}$.

The issue is therefore not on the origin of the volatiles on Earth but on their evolution and redistribution when asteroids and comets collided on the Hadean Earth.

\section{1. 2. Chemical composition of the early Earth atmosphere at late Hadean}

The formation and composition of the secondary atmosphere formed during the Hadean relied on several complementary transformations of the volatiles contained in the impactors reaching the Earth during that geological eon.

\section{1. 2. 1. Direct volatiles release from impactors in early Hadean}

The release of volatiles on Earth from impactors, namely asteroids, interplanetary dust, and possibly comets during early Hadean, basically depended on the size and the velocities of the impactors ${ }^{9}$. Larger impactors delivered larger contents of water and organic species on Earth, but with the size increased also the energy of the shock. With large impactors, organics were partially or totally released in the atmosphere, but in chemical forms far from the complex organic molecules identified in carbonaceous meteorites, such as in the Orgueil and in the Murchison meteorites ${ }^{10,11}$. When extrapolating the present-day background bombardment flux on the Moon, aside from the possible further intense contribution of a Late Heavy Bombardment, the absolute contribution of impactors in the volatile budget of the early Earth globally decreased by several orders of magnitudes between early Hadean and late Hadean ${ }^{12}$. Volatiles were provided in abundance during the massive bombardment events that occurred during early Hadean, mostly under degraded forms, releasing water steam and degraded organic molecules in the proto-atmosphere of the early Earth. $\mathrm{CO}_{2}$ is predicted to be the major $\mathrm{C}$-containing molecule released in the atmosphere after heating the incoming carbonaceous materials ${ }^{13}$.

\section{1. 2. 2. Ingassing during early Hadean}

After the giant impact leading to the formation of the Moon $\sim 4.47 \mathrm{Ga}$ ago, the Earth massively melted. The numerous and massive impacts during early Hadean also contributed to generate a dense steam atmosphere with a high greenhouse effect ensuring the sustainable melt of the Earth mantle ${ }^{14}$. Between 4.47 and $4.3 \mathrm{Ga}$ in the Hadean, the Earth was therefore covered by a deep convecting magma ocean ${ }^{15}$. This melting enabled an evolution of the Earth mantle, with a loss of molten iron towards the Earth's core ${ }^{16}$. The redox state of the mantle changed becoming more oxidative along with the metallic iron loss. Geochemical analysis of old igneous rocks 
showed that the redox state of the Earth mantle is oxidative and stable since at least $3.8 \mathrm{Ga}^{17,18}$. This would also be in favour of rather oxidative conditions of the mantle during late Hadean. Yet the rocky ocean trapped and accumulated volatiles from the proto-atmosphere through thermodynamical gas solubility equilibrium. Volatiles were "digested" in the magma ocean according to the mantle redox state and their respective solubility properties. Therefore volatiles, which were trapped in the mantle during the previous magma ocean period, were probably totally oxidized.

With the decrease of the surface temperature, the magma ocean progressively froze and water vapour condensed to form the first oceans. The degassing of the mantle through volcanism and the further exchanges of the atmosphere with the nascent oceans then largely controlled the atmospheric content during late Hadean.

[Insert Figure 4.1 here]

\section{1. 2. 3. Release by volcanism in late Hadean and further exchanges with the nascent oceans}

Volcanism released oxidized volatiles from the Earth mantle, enriching the atmosphere with large amounts of $\mathrm{CO}_{2}, \mathrm{~N}_{2}$ and $\mathrm{H}_{2} \mathrm{O}$, and possibly marginally $\mathrm{CO}$ and $\mathrm{H}_{2}^{8} \cdot \mathrm{N}_{2}$ is a very inert species, and its amount is expected to be rather similar since the Hadean period as today, with an order of magnitude of $\sim 1$ bar. On the contrary $\mathrm{CO}_{2}$ atmospheric abundance evolved significantly with its interaction with the nascent oceans. From a partial pressure of $\sim 100$ bars in the atmosphere, $\mathrm{CO}_{2}$ abundance decreased rapidly. $\mathrm{CO}_{2}$ went indeed efficiently dissolved in water and transformed into calcium carbonates by reaction with calcium silicates on the seabed. Subduction of the oceanic crust finally buried carbonates, indirectly returning back the degassed $\mathrm{CO}_{2}$ into the Earth mantle ${ }^{19}$. The age of the carbonates cycle is currently debated but it could have had a rapid onset, possibly within less than 10 million years ${ }^{20}$.

With the decrease of the $\mathrm{CO}_{2}$ concentration, the greenhouse effect of the early Earth atmosphere was consequently weakened and the Earth surface temperature decreased accordingly. The remaining $\mathrm{CO}_{2}$ abundance in the atmosphere is not precisely constrained as no remaining rock from Hadean subsists ${ }^{3}$. But as liquid water was present and persisted, the early Earth climate during late Hadean and early Archean was habitable, imposing flexible constraints on $\mathrm{CO}_{2}$ as main atmospheric greenhouse gases in this stage. The upper limit consistent with the presence of liquid water at late Hadean is $\sim 10$ bar for $\mathrm{CO}_{2}$ partial pressure ${ }^{21}$, leading to a hot resulting surface temperature of $80-90^{\circ} \mathrm{C}^{22}$. The oldest determination of $\mathrm{CO}_{2}$ partial pressure obtained from archean rocks of $3.2 \mathrm{Ga}$ provides a lower limit of a few $\mathrm{mbar}^{23}$. 


\section{1. 2. 4. Methane contribution at late Hadean}

The uncertainty on $\mathrm{CO}_{2}$ concentration during late Hadean is also driven by the effect of the complementary efficient greenhouse gas that contributed to the early Earth habitable climate since late Hadean: methane $\mathrm{CH}_{4}$. Two cumulated sources of methane are considered before the emergence of life on the early earth: its formation by serpentinization beneath the ocean floor followed by degassing from mid-ocean ridges, and further contributions from meteoritic impacts. At the lower temperature of the habitable late Hadean, the impact of $\mathrm{Cl}$ carbonaceous chondrites would contribute to $\mathrm{CH}_{4}$ degassing ${ }^{8}$. Methane was therefore possibly non negligible on the early Earth with concentrations of about few parts per millions $\left(\mathrm{ppm}_{\mathrm{v}}\right)$, but uncertainties on its concentrations remain important ${ }^{24}$.

\section{1. 3. Summary of the atmospheric composition on the early Earth supporting}

\section{the emergence of life}

When life appeared during late Hadean or early Archean, the Earth was by definition already habitable. Water oceans were formed, constraining the Earth surface temperature roughly between between 0 and $150^{\circ} \mathrm{C}$, depending on the 1-10 bar surface pressure. The Earth Hadean climate remains however uncertain within this broad window, due to lack of geological evidence on the precise atmospheric composition, which controlled the greenhouse effect at that eon. Water vapour was present in the atmosphere, but in absence of ozone layer, no tropopause existed yet to trap water in the first kilometres of the atmosphere as today. The atmosphere was possibly already $\mathrm{N}_{2}$-based, or with comparable contributions of $\mathrm{N}_{2}$ and $\mathrm{CO}_{2}$, leading to a total pressure at the surface of 1-10 bar. Methane traces of a few $\mathrm{ppm}_{\mathrm{v}}$ were additionally provided by meteoritic impacts and serpentinization. 


\subsection{Early Earth photochemistry}

\section{2. 1. Atmospheric photochemistry triggered by the young Sun}

\section{2. 1. 1. Importance of the UV spectral range}

The major source of energy deposited in the atmosphere comes from solar radiation. Depending on the wavelength of the photons, solar radiation can even break bonds of the molecules composing the atmosphere, triggering atmospheric photochemistry.

For instance the bonding energy of the simple $\mathrm{C}-\mathrm{H}$ bond is $\mathrm{D}_{\mathrm{C}-\mathrm{H}}=410 \mathrm{~kJ} \mathrm{~mol}^{-1}(4.25$ $\mathrm{eV}$ ). For a solar photon, this would correspond to a $\sim 290 \mathrm{~nm}$ photon energy, in the UV range. To dissociate the very strong triple bond of $N_{2}$, the energy required is higher with $D_{\mathrm{N} \equiv \mathrm{N}}=945 \mathrm{~kJ} . \mathrm{mol}-1(9.79 \mathrm{eV})$, involving photons with wavelengths lower than $126 \mathrm{~nm}$, in the Vacuum-UV range. Within the solar spectrum, the wavelengths enabling photochemistry are therefore related to the UV range.

The early Earth atmosphere was submitted to the radiation of the young sun, which was about $30 \%$ less luminous than today ${ }^{25}$, but with a stronger X-ray and UV contributions $^{26}$. The photochemistry occurring on the early habitable Earth was therefore even more efficient than today.

\section{2. 1. 2. Low concentrations with large atmospheric impacts}

Compared to the oceans, the atmosphere is a diluted matter. The concentration unit commonly used in solution, the number of moles per litre $\left(\mathrm{mol} . \mathrm{L}^{-1}\right)$, is not adapted for describing much lower atmospheric concentrations. Two kinds of concentrations are used: absolute concentrations with the number of molecules per cubic centimetre $\left(\right.$ molec. $\mathrm{cm}^{-3}$ ), and relative concentrations, expressed in mole fractions without units.

For an estimated surface pressure of the early Earth of about 1 bar, the order of magnitude of the total atmospheric concentration would be $10^{19}$ molec. $\mathrm{cm}^{-3}$. This number mostly accounts for the main constituents composing the early Earth atmosphere: $\mathrm{N}_{2}$ and $\mathrm{CO}_{2}$. $\mathrm{CH}_{4}$, as a minor compound, will preferentially be expressed in mole fraction. Thus methane accounts for a few $\mathrm{ppm}_{\mathrm{v}}$ of the total atmospheric concentration of the early Earth. Despite this apparently low concentrations compared to $\mathrm{N}_{2}$ and $\mathrm{CO}_{2}$, the atmospheric impact of this minor component is central. It is indeed an excellent absorber of the intense Lyman-alpha UV emission line of the sun. Its photolysis in the atmosphere of Titan, the largest satellite of Saturn, leads for instance to the formation of a permanent photochemical smog with strong impact on the chemistry and the climate of this frozen satellite ${ }^{27}$.

As illustrated on methane, the atmospheric impact of minor compounds can be very high, both on the climate and on the prebiotic potential of the species formed by photochemistry on the early Earth. Atmospheric photochemistry is a chemistry of traces. The issue is to appreciate the formation and impact of all the trace molecules that were generated by atmospheric photochemistry in the early Earth.

\section{2. 1. 3. Photolysis from the upper atmosphere down to the surface}


Atmospheric chemistry involves complex chemical networks of reactions occurring all at the same time, but with various efficiencies depending on the local environmental conditions (basically solar radiation, temperature, pressure) and on the abundance of the atmospheric constituents. As these conditions vary drastically with altitude, chemical networks also evolve with altitude and the relative abundance of chemical constituents on the early Earth changed significantly between the surface and its upper atmosphere.

The upper atmosphere was submitted to the full radiation of the young Sun, but the deeper atmospheric layers receive only the remaining radiations after absorption by the overhead atmospheric layers. This altitude dependent photon flux deposition into the atmosphere leads to processes specific of the highly energetic UV photons available in the spectrum at a given altitude. Photodissociation and photoionization of the main atmospheric constituents, $\mathrm{CO}_{2}, \mathrm{~N}_{2}, \mathrm{H}_{2} \mathrm{O}$, and $\mathrm{CH}_{4}$, trigger the atmospheric chemistry at different altitudes. As a rough first approximation, we can consider that the energetic extreme ultraviolet photons are absorbed at high altitudes by the most stable and abundant components of the atmosphere, leading to a possible partial ionization of the atmosphere. The less energetic UV photons penetrate at lower altitudes and photolyse more fragile and less abundant atmospheric molecules.

$\mathrm{N}_{2}$ is a particularly inert component, which requires extreme ultraviolet photons to be dissociated and ionized. Its direct reactivity is therefore limited to high altitudes in the early Earth atmosphere, namely the thermosphere.

Its ionization threshold is at $\lambda=80 \mathrm{~nm}$ :

$$
N_{2}+h v \rightarrow N_{2}^{+}+e^{-},
$$

And its dissociation threshold occurs at $\lambda=102 \mathrm{~nm}$ :

$$
N_{2}+h v \rightarrow N(4 S)+N(2 D)
$$

$\mathrm{CH}_{4}$ ionization threshold is at the wavelength of $98 \mathrm{~nm}$, and $\mathrm{CH}_{4}$ is also a strong absorber of Lyman- $\alpha$ radiations $(\lambda=121.6 \mathrm{~nm})$, an intense emission line in the solar spectrum. This rather stable molecule is therefore photoionized and dissociated also at rather high altitudes. The photoproducts from methane with Lyman- $\alpha$ radiations have been well established experimentally ${ }^{28,29}$, with fragment quantum yields $\Phi_{\mathrm{i}}$ :

$$
\begin{aligned}
& \mathrm{CH}_{4}+h v \rightarrow \mathrm{CH}_{3}, \Phi_{1}=0.42 \\
& \mathrm{CH}_{4}+h v \rightarrow \mathrm{CH}_{2}, \Phi_{2}=0.51 \\
& \mathrm{CH}_{4}+h v \rightarrow \mathrm{CH}, \Phi_{3}=0.07
\end{aligned}
$$

$\mathrm{CO}_{2}$ is photoionized with photons at wavelengths lower than $90 \mathrm{~nm}$, occurring in the thermosphere, but its photodissociation requires much less energetic UV photons with $\lambda<204 \mathrm{~nm} . \mathrm{CO}_{2}$ photolysis extends down until the upper troposphere. $\mathrm{CO}_{2}$ is thus is an important source of free oxygen atoms almost everywhere in the atmosphere according to the following photolysis reaction: 


$$
\mathrm{CO}_{2}+\mathrm{hv} \rightarrow \mathrm{CO}+\mathrm{O}
$$

$\mathrm{H}_{2} \mathrm{O}$ photodissociation threshold is at $\lambda=240 \mathrm{~nm}$. This moderate UV wavelength enables the photodissociation of water to occur until the Earth surface ${ }^{30}$. Water photolysis was a major source of oxidative $\mathrm{OH}$ radicals in the early Earth atmosphere:

$$
\mathrm{H}_{2} \mathrm{O}+\mathrm{hv} \rightarrow \mathrm{OH}+\mathrm{H}
$$

\section{2. 1. 4. Atmospheric photoproducts as key prebiotic precursors: $\mathrm{HCN}$ and $\mathrm{HCHO}$}

Radicals and ions are produced from the photolysis reactions described above. These reactive species collide and recombine rapidly with neighbour molecules following bimolecular or termolecular processes depending on the local pressure. Some of these further processes are known to produce some key prebiotic molecules in the atmosphere of the early Earth: hydrogen cyanide HCN and formaldehyde $\mathrm{HCHO}$. Their prebiotic interest is explained later, in section X.3.

Carbon monoxide (CO) produced by the photolysis of $\mathrm{CO}_{2}$, reacts with hydrogen atoms produced in abundance by water photolysis, thus forming the formyl radical $\mathrm{HCO}$. Formyl radical can recombine with itself to form formaldehyde $\mathrm{HCHO}$ :

$$
\begin{gathered}
\mathrm{CO}+\mathrm{H} \rightarrow \mathrm{HCO} \\
\mathrm{HCO}+\mathrm{HCO} \rightarrow \mathrm{HCHO}+\mathrm{CO}
\end{gathered}
$$

$\mathrm{HCN}$ comes from reactions coupling $\mathrm{N}_{2}$ and $\mathrm{CH}_{4}$ photolysis. It could have been of particular importance during the hadean and early archean eons ${ }^{31}$. The main reaction sequence is the following: $\mathrm{N}(4 \mathrm{~S})$ nitrogen atoms adds with $\mathrm{CH}_{3}$ radical. Then $\mathrm{H}_{2} \mathrm{CN}$ is stabilized into the $\mathrm{HCN}$ molecule after an $\mathrm{H}$-loss process.

$$
\begin{gathered}
\mathrm{N}(4 \mathrm{~S})+\mathrm{CH}_{3} \rightarrow \mathrm{H}_{2} \mathrm{CN} \\
\mathrm{H}_{2} \mathrm{CN}+\mathrm{H} \rightarrow \mathrm{HCN}+\mathrm{H}_{2}
\end{gathered}
$$

Past photochemistry in the prebiotic atmosphere is deeply explored through photochemical modelling $8,31-33$. However despite the thousands of reactions already known and implemented in the photochemical models, processes leading to complex molecules with more than five heavy atoms are still largely unknown. To further and explore the past photochemistry that occurred on the early Earth, experimental simulations have been develop through decades to mimic in the laboratory the atmospheric chemical systems that could have occurred on the early Earth.

\section{2. 2. Experimental simulation of the Early Earth photochemistry.}




\section{2. 2. 1. From theory to laboratory simulation}

In the $19^{\text {th }}$ century, Pasteur demonstrated through a series of experiments the nullity of the theory of apparition of life through spontaneous generation that wrongfully dominated discussions on this topic since Aristotle ${ }^{34}$. However, around the same time, Darwin theory on the evolution of species demonstrated the existence of a common ancestor, that must have had appeared on the early Earth. In his book Origin of Life, Alexander Oparin was the first to propose a possible - and theoretically testable - hypothesis to explain the discrepancy between the fact that life must have had appeared at an undefined moment in the past on Earth, and yet that it was not spontaneously generated from inanimate matter at the present days. For further reading, we strongly advise the interested reader to refer to Oparin's book, truly ground-breaking for its time and where many of the hypothesis he formulated are still being investigated to this day.

Oparin suggested that the environment of the early Earth may have been significantly different from the one of modern Earth, and provided conditions more favourable for the apparition of life. In particular, Oparin discussed that the organic material needed to evolve from pure chemistry to the emergence of life may have been externally delivered, either through the influx on Earth of organic compounds from meteorites, or through an endogenous formation in Earth primitive atmosphere $^{35}$. The concept of abiogenesis was further extended by Jack Haldane who first introduced the theory of primordial soup in $1929^{36}$.

While Oparin and Haldane never experimentally tested their ideas, this was first done in the early 50's (Oparin's originally published his ideas in Russian in 1924 but was only translated to English in 1938). First experiments by Wilde et al. exposed mixtures of carbon dioxide and water, contrary to Oparin's point of view who considered that the atmosphere of the early Earth was reducing, to high frequency electric arc, "in the hope of isolating 1 and possibly 2 carbon-atom compounds [...] of special interest in relation to the basic photosynthesis process and also carries implication with respect to the origin of living matter on earth." ${ }^{37}$. However, as stated by the authors themselves, these experiments failed "to establish the presence of any interesting reduction products in a reproducible way." Almost concomitantly to Wilde's experiment, was submitted to the same journal a paper by a graduate student, Stanley Miller, that would pave the road for more than half a century of laboratory experiments on the early Earth.

\section{2. 2. 2. The Miller-Urey Experiment}

Following his Similan Lectures at Yale University, the Nobel prize awardee Harold Urey published in 1952 The Planets: Their Origin and Development in which he exposed, amongst many other, his theories on the composition of the early Earth atmosphere $^{38}$. Urey considered that the early - a period then-defined by beyond 2 Gyr - atmosphere was extremely reduced and composed principally of ammonia, methane and hydrogen. Following his lectures, Miller approached Urey to suggest a thesis project on an experimental approach to test Urey's theories. For a more descriptive story of Miller and Urey personal interactions leading to this work, we strongly encourage reader to refer to Lazcano and Bada $2003^{39}$. 
Miller proposed a simple, yet elegant, experimental design to simulate prebiotic chemistry in the atmosphere. A gas mixture of methane, hydrogen, ammonia and water was flowed a recirculated through an electric discharge. Regarding the energy source and contrary to a very common belief in the community, Miller did not try to simulate lightning in the early Earth atmosphere but simulated the energy influx from the sun by using an electric discharge instead of an ultraviolet light, "because quartz absorbs wavelengths short enough to cause photo-dissociation of the gases" 40 . This limitation still stands to this day and explains why most of the dozens experiments that followed to simulate early Earth photochemistry used a plasma setup and not an UV lamp.

After passing through the discharge, the gas phase containing the original reactive and the newly formed products would interact with liquid water. This design induced an accumulation of compounds produced in the discharge into the water phase. Meanwhile $\mathrm{NH}_{3}, \mathrm{CH}_{4}, \mathrm{H}_{2} \mathrm{O}$ and $\mathrm{H}_{2}$, more volatile, were able to recirculate through the system.

Miller published a very succinct report on his experiment in 1953 where he stated that after running continuously the experiment for a week, the water went from transparent to pink to end up forming a dark and turbid red solution by the end of the week. This colour was due to complex organic molecules adsorbed on the silica. $\mathrm{He}$ also noted the presence of insoluble organic material. Chromatographic analyses revealed the formation of amino acids, in particular Glycine and $\alpha$ - and $\beta$-alanine (See Figure 2 in Miller 1953).

In Miller and Urey 1959 paper ${ }^{41}$, they stated further analysis of the products of the 1953 experiments. Re-analysis using modern technology was also carried on in 2008 and 2011 on unopened vials including on previously unreported simulation including sulphur compounds ${ }^{42,43}$.

In total, all these analyses allowed the detection of 42 different amino acids in Miller's extract ( 7 were detected originally by Miller himself using paper chromatography), together with a wide variety of organic compounds including amines, acids, aldehydes, ketones. This demonstrates the wide variety and complexity of the chemical network at act in such a simple experiment.

\section{2. 2. 3. Beyond the pioneers' work, current advances in laboratory simulations}

Miller's work opened a wide new field of investigation on laboratory simulation of not only the early Earth environment. These subsequent works can be divided into two main categories: global simulations, as Miller's, attached to reproduce a system and analysed end-results without a priori; and targeted simulations, looking at a specific process leading to the formation of a prebiotic molecule. Obviously, both approaches have their advantages and inconvenient and have provided tremendous amount of information that could not be simply summarized in a book chapter. The targeted approach has the advantage to be able to strictly elucidate one or a few chemical pathways for specific. This was used for example from the 70 ' to the early 2000 ' to 
identify key organic component for prebiotic synthesis such as hydrogen cyanide and formaldehyde (see section X.3.2).

Regarding global simulations, the field rapidly stirred away from the early Earth environment to simulate planetary atmospheric chemistry. This was due partly to advances regarding the composition of the early Earth atmosphere, tending to demonstrate a completely oxidized atmosphere on Earth during the late Hadean and proto Archean. Another factor was probably the development of planetary exploration space missions that within a few decades returned more information on these environments that we could ever get on the early Earth. A particular sub field bloomed on the study of Titan atmosphere. Titan is the largest satellite of Saturn and the only one in the solar system to have a substantial atmosphere (1.5 bar ground pressure). In addition, this atmosphere is highly reducing, being comprised of molecular nitrogen $(\sim 98 \%)$ and methane $(\sim 2 \%)$, and thus highly favourable to host complex organic chemistry as demonstrated in Miller's experiment.

While not being directly applicable to the early Earth, the results obtained during this period motivated the development of a new generation of experimental setup, paving the road for a "return" to early atmospheric laboratory experiment. In particular, these allowed to correct some of the limitations of Stanley Miller's setup such as the energy source used or not simulating an open atmosphere but rather a closed circuit. An interested reader should refer to the review by Cable et al. $2012^{44}$. Amongst all experimental work done since the Miller era, a noticeable one was done by a research group at NASA Ames research centre lead by Bishnun Khare and Carl Sagan. They reported the formation of solid spherical organic material, and coined the term tholins for it, from irradiation of gaseous mixture ${ }^{45}$. Such complex organic aerosol would form in a reducing atmosphere and subsequently deposit onto the surface where it could interact with liquids.

From the early 2000s, recent advances in our knowledge of the early Earth atmosphere and its partially reducing capacities (see part 1.2 of this chapter) sparked new interest. Laboratory simulations of the early Earth atmosphere photochemistry were restarted using atmospheric gas mixtures diverging greatly from the Miller-Urey experiment. These experiments consider early Earth atmosphere to be in an oxidative state but with an amount of reducing compounds significant enough to trigger organic chemistry.

This reducing component could have been methane, which could be justified by the fact that no geological record support high level of $\mathrm{CO}_{2}$ in the early Archean ${ }^{46}$. In their experiment, Trainer et al. 2004 irradiated mixture of $\mathrm{N}_{2}, \mathrm{CO}_{2}$ and $\mathrm{CH}_{4}$, exploring various $\mathrm{CH}_{4} / \mathrm{CO}_{2}$ ratios. They report the formation of organic particles with only 1,000 ppmv $\mathrm{CH}_{4}$ concentration. They note the importance of the $\mathrm{C} / \mathrm{O}$ ratio on the formation of organic material in the atmosphere, but that atmospheric organic synthesis could be sustainable up to a concentration of $\mathrm{CO}_{2}$ in the atmosphere of $5 \%$. For further reading on the different scenario of early Earth atmospheric composition that have been explored experimentally, we refer the reader to the review of Trainer et al. 2013 47 . 
The latest tested hypothesis regarding a reducing component is it inclusion at high altitude under the form of molecular hydrogen. Fleury et al. $2017^{48}$ experimentally tested a hypothesis suggesting that $\mathrm{H}_{2}$ could have been present in large amount in the early Earth upper atmosphere (ionosphere) due to water photolysis and degassing ${ }^{32}$. Their experimental setup is presented in Figure X.2. In their experiment they used $\mathrm{CO}_{2}$ as the sole carbon source, in a mixture with $\mathrm{N}_{2}$ and $4 \% \mathrm{H}_{2}$. They observed gaseous reactivity leading for example to the formation of ammonia, and to the formation of Nitrogen-bearing solid organic particles bearing. They suggest that such organic aerosols could have formed in the early Earth ionosphere and subsequently deposited at the surface, providing an efficient source of prebiotic material on the early Earth.

\section{[Insert Figure 4. 2 here]}

Although one cannot reproduce with absolute certainty in the laboratory the atmosphere of the early Earth, exploring the parameter space of both gaseous composition and energy source over the past 30 years has been extremely insightful. In particular, it is now clear that the vast majority of composition scenarii enable the onset of an active atmospheric photochemistry, as soon as the atmosphere has an even minor reducing component.

Although the temporal and spatial extend of this phenomenon is still unknown, it now appears clearly that such photochemistry on the early Earth could have had strong prebiotic influence. It induced the formation of secondary volatiles compounds able to influence atmospheric composition and radiative balance, as well as the formation of more complex macromolecules forming organic aerosols. Such organic aerosols would have subsequently deposit onto, and interact with, the surface and its water content, bringing the necessary building blocks of life needed for the formation of a primordial soup as theorized almost a hundred years ago by Alexander Oparin. 


\subsection{Prebiotic potential of atmospheric chemistry on the early Earth}

\section{3. 2. Prebiotic molecules}

The formation pathways of most of the detected compounds in laboratory experiments, from Miller's to more recent ones, still remain to be elucidated. It is thought that many, if all, of them require two major first-step produces: hydrogen cyanide and formaldehyde. Miller originally thought that the principal mechanism of formation of amino acids in his experiments passed through the Strecker reaction due to reaction of $\mathrm{HCN}$ with ammonia and an aldehyde, forming an aminonitrile followed by its hydrolysis into an amino acid.

However, such mechanism would require the formation of a large variety of aldehyde at high concentration in the experiment, which has not been demonstrated. Another pathway was later proposed by Matthews to explain amino-acids formation through hydrogen cyanide polymerisation ${ }^{49}$. This hypothesis has been widely debated, mainly because such a polymer would require a high level of self-organization, poorly consistent with such complex chemistry.

A more modern approach still considers $\mathrm{HCN}$ to be a key component of prebiotic chemistry leading to the formation of amino acid, but through the presence of $\mathrm{HCN}$ oligomers, which have been shown to release a large quantity of amino-acids under hydrolysis. For a more detailed discussion on this debate and schematic of key reactions, we refer the reader to Raulin $2005^{50}$ and reference therein. In all cases, it appears that hydrogen cyanide and formaldehyde both play a key role in the chemistry happening in laboratory simulation of the early Earth. Their formation pathway can be elucidated as presented in section X.2.1.4 of this chapter.

The formation of the first bricks leading to prebiotic molecules, such as hydrogen cyanide or formaldehyde or even more complex such as amino-acids, as now been elucidated and demonstrated to have been possible in the early Earth atmosphere. However, the fate of these organic molecules at the surface of the early Earth, where they could interact with liquid water, still remains to be fully investigated. Numerous investigations have already looked into this issue, and it goes beyond the scope of this chapter to review them. A brief summary of these experiments is that we can now explain through hydrolysis of organic matter formed in the atmosphere the formation of amino-acids and assemble them into peptides, and the one of puric bases. Open questions remain on the formation of sugars - where formaldehyde likely plays an important role -, nucleotides and membranes.

A final point on prebiotic material of atmospheric origin, as we know, life today favors enantiomeric excess. However, all the reaction described above form racemic amino-acids, as demonstrated by Khare and Sagan in $1981^{45}$. While self-replicating mechanisms in aqueous solution can explain the amplification of small original racemic excess, the origin of this excess remains to be elucidated for atmospheric organic material. 


\section{3. 3. Climate impact}

\section{3. 3. 1. Water, clouds and greenhouse gases}

Beyond the prebiotic interest of the products themselves, the atmosphere and its content also strongly influenced the surface temperature of the early Earth. When life appeared between late Hadean and early Archean the Earth hosted its first oceans and continents. The surface temperature could have been mild or hot depending on the abundances of $\mathrm{CO}_{2}$ and $\mathrm{CH}_{4}$, as both are efficient greenhouse gases.

Water, as a greenhouse gas and a source for clouds, also deeply contributed to the global climate of the early Earth. As shown with a global climate 3D model taking into account the formation of tropospheric clouds and their radiative effect, ice-free water ocean belts could have been maintained between the tropics and the equator during the early Archean with only 1 mbar of $\mathrm{CO}_{2}$ and no methane ${ }^{51}$. Furthermore, photochemistry of $\mathrm{H}_{2} \mathrm{O}$ at low altitude in the troposphere further influenced the chemistry in the upper atmospheric layers and so far the early Earth climate. Thus water photolysis at low altitude produced $\mathrm{H}$ atoms that partially recombined into $\mathrm{H}_{2}$ molecules. $\mathrm{H}$ and $\mathrm{H}_{2}$ are light molecules that diffused vertically more rapidly than $\mathrm{CO}_{2}$ and $\mathrm{N}_{2}$, so that the upper atmosphere was progressively enriched in $\mathrm{H}_{2}$ with altitude. Then at high altitude, the coupled photochemistry of $\mathrm{CO}_{2}$ and $\mathrm{H}_{2}$ led to a reformation of water at high altitude through the following mechanism ${ }^{52}$ :

$$
\begin{gathered}
\mathrm{CO}_{2}+\mathrm{hv} \rightarrow \mathrm{CO}+\mathrm{O} \\
\mathrm{O}+\mathrm{H}_{2} \rightarrow \mathrm{OH}+\mathrm{H} \\
\mathrm{OH}+\mathrm{H}_{2} \rightarrow \mathrm{H}_{2} \mathrm{O}+\mathrm{H}
\end{gathered}
$$

The formation of water at high altitude, low pressure and low temperature resulted in water condensation and the formation of water ice clouds comparable to present polar stratospheric or mesospheric clouds ${ }^{53,54}$. Climate impact of high altitude water ice clouds on the early Earth is not well constrained and depends on their optical thickness, with cumulated effects on infrared radiation at high altitudes (warming effect) and on the albedo in the visible wavelength range (cooling effect). Another important uncertainty on the early Earth climate is moreover the abundance and optical properties of photochemical aerosols.

\section{3. 3. 2. Photochemical aerosols}

Direct radiative effect of dry aerosols. Atmospheric haze can drastically affect the radiative balance of atmospheres. Depending on the size and the intrinsic spectral properties of aerosols, light scattering and absorption occur, modifying the local temperature of the atmosphere and the radiation balance towards the surface. On Titan, the large amount of UV absorbing aerosols high in the atmosphere (above 300 $\mathrm{km}$ ) leads to an increase of the local temperature by about $10 \mathrm{~K}$. As they scavenge UV radiation at high altitudes, aerosols also prevent a large part of the UV radiation to reach the surface and lead to a cooling of the surface temperature, also by about $10 \mathrm{~K}^{55}$. On present-Earth, secondary organic aerosols are mainly produced close to 
the surface due to the abundant organic emissions from the ground. Their UV absorption properties lead in the opposite to a warming effect close to the surface. Depending on the altitude of formation and their absorption properties, organic aerosols increase or decrease the surface temperature of the early Earth. The radiative effect of the aerosols is based on the determination of their optical indices, predicting in particular the UV absorption properties of the aerosols. The UV absorption band of organic aerosols, in terms of position and intensity, is strongly depending on the chemical composition of the aerosols, the incorporation of nitrogen and oxygen in the organic structure leading to higher UV absorption properties ${ }^{56-58}$. Experimentally, photochemical aerosols representative of early-Earth atmosphere were shown to be less efficiently produced at high $\mathrm{CO}_{2} / \mathrm{CH}_{4}$ ratio ${ }^{46}$, but with larger UV absorption properties ${ }^{56}$.

Indirect climate effect with aerosols acting as cloud condensation nuclei. The formation of aerosols at high altitudes through photochemistry even in the absence of methane ${ }^{48}$ triggered an important microphysical process: aerosols when sedimenting down to the surface entered atmospheric layers where water condense. Aerosols act as cloud condensation nuclei, with an important effect on the radiative balance of the early Earth atmosphere. The effect of clouds on the radiative balance of Earth is far from simple. Two major opposite effects are nowadays relatively well accepted. High cirrus clouds made of ice crystals are too thin to reflect significantly solar UV radiation and will mainly act as a warming greenhouse element, scattering back to Earth infrared telluric radiation. In the opposite, low stratocumulus clouds made of liquid water droplets are much thicker and contribute globally to decrease the UV flux reaching the surface, and finally cool the Earth surface. The net effect of clouds has still uncertainties but is globally negative, with a net effective cooling of the Earth surface ${ }^{59}$. High altitude photochemical aerosols therefore provided a cooling effect acting as cloud condensation nuclei by interaction with liquid water, possibly in favor of stable liquid water at the Earth surface. This aerosol effect is however still poorly constrained and addresses an open issue on the early climate of the Earth. 


\subsection{Conclusion and perspectives: what do we learn from other planets}

The emergence of life occurred on a favourably habitable planet that was the early Earth 4.3 to $3.7 \mathrm{Ga}$ ago. In synergy with the oceans, the atmosphere ensured invaluable environmental habitability conditions to sustain the emergence and durability of the first forms of life. Atmospheric photochemistry actually contributed to a reasonably non-hostile climate and could also have provided prebiotic organic materials to the oceans at a large global scale. Several key parameters were highlighted such as the $\mathrm{CO}_{2} / \mathrm{CH}_{4}$ ratio, but also the effect of chemistry at high altitudes that triggers nitrogen chemistry with extreme UV radiations. However these precise effects are approximated and reconstructed at present days through experimental simulations and modelling as no more geological record exists to inform us on the atmospherical content at these early stages of the Earth. This lack of direct observables on the early Earth is partially answered through the search for proxies of the early-Earth in the solar system and beyond. Titan, the largest satellite of Saturn is an interesting extreme case with a dense atmosphere made of nitrogen and methane. This atmospheric "organic factory" 60 produces a permanent organic photochemical smog that is predicted to contain prebiotic molecules ${ }^{61}$. Titan was one of the main scientific targets of the Cassini-Huygens space mission ${ }^{62}$ that successfully orbited Saturn between 2004 and 2017 and will be further explored in the future with the Dragonfly space mission ${ }^{63}$ that will be launched in 2026 . The search for Earth-like planets in exoplanetary systems is another inspiring perspective to learn about our past. The NASA James Webb and ESA-ARIEL space telescopes are in this frame expected to provide pioneer information on their atmospheres, which in return could help us better constrain the atmospheric chemistry on the early Earth. 
FIGURE AND TABLE CAPTIONS

Figure 4. 1 Historical timeline and formation of the early Earth atmosphere between the Hadean and the Archean eons.

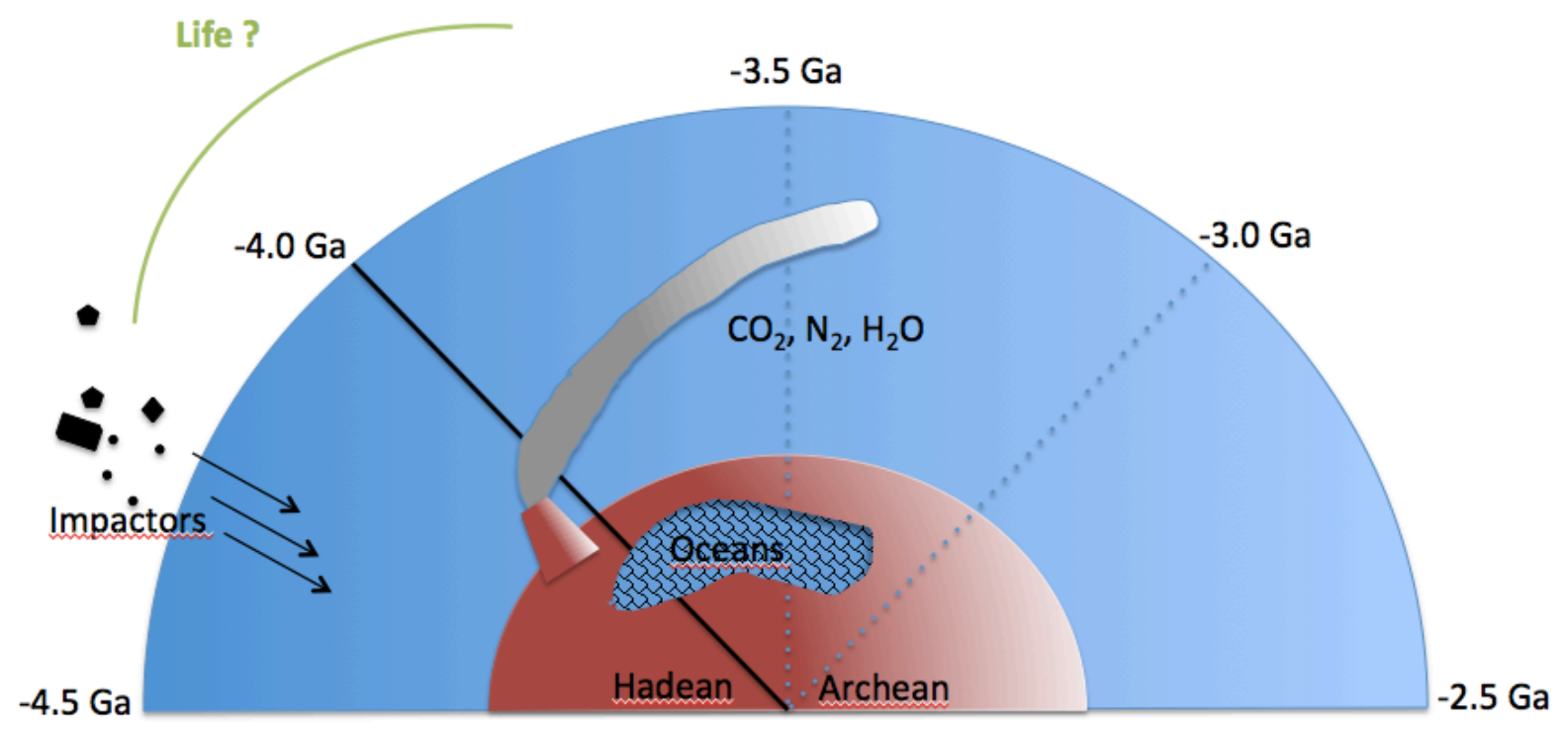


Figure 4.2: Picture of the setup used by Fleury et al. to investigate ionosphere formation of organic aerosols in the early Earth. This setup is an example of the small dozen modern setup currently operating for this kind of laboratory investigations. With permission of LATMOS-UVSQ.

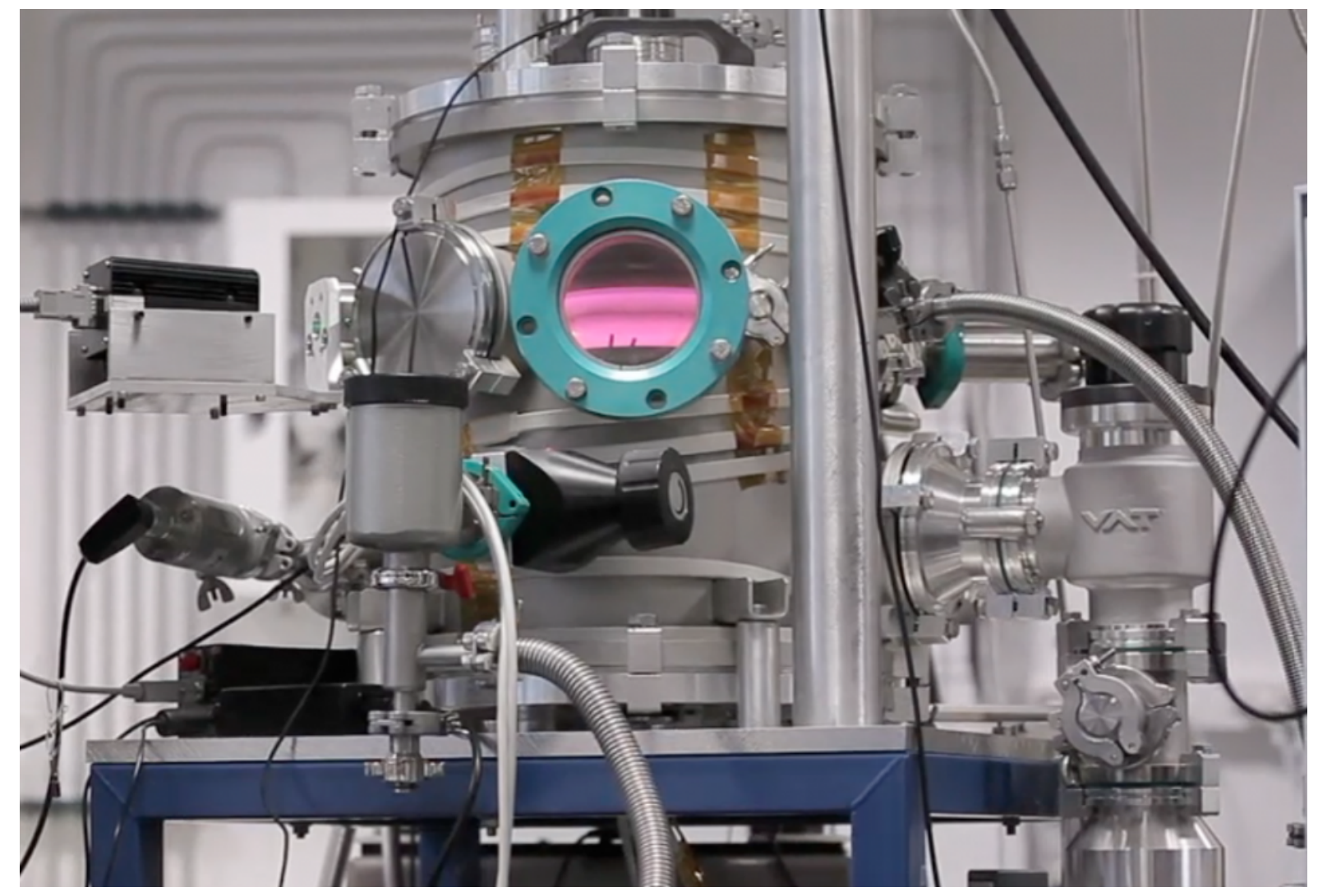




\section{ACKNOWLEDGEMENTS}

N.C. thanks the European Research Council for funding via the ERC PrimChem project (grant agreement No. 636829). T.G. thanks the Programme National de Planétologie for funding.

\section{References}

1. A. P. Nutman, V. C. Bennett, C. R. L. Friend, M. J. Van Kranendonk and A. R. Chivas, Nature, 2016, 537, 535-538.

2. S. J. Mojzsis, T. M. Harrison and R. T. Pidgeon, Nature, 2001, 409, 178-181.

3. S. A. Bowring and I. S. Williams, Contributions to Mineralogy and Petrology, 1999, 134, 3-16.

4. D. Schulze-Makuch, A. Mendez, A. G. Fairen, P. von Paris, C. Turse, G. Boyer, A. F. Davila, M. R. d. S. Antonio, D. Catling and L. N. Irwin, Astrobiology, 2011, 11, 1041-1052.

5. H. Lammer, J. H. Bredehöft, A. Coustenis, M. L. Khodachenko, L. Kaltenegger, O. Grasset, D. Prieur, F. Raulin, P. Ehrenfreund, M. Yamauchi, J. E. Wahlund, J. M. Grießmeier, G. Stangl, C. S. Cockell, Y. N. Kulikov, J. L. Grenfell and H. Rauer, The Astronomy and Astrophysics Review, 2009, 17, 181-249.

6. K. Lodders, The Astrophysical Journal, 2003, 591, 1220-1247.

7. A. Morbidelli, J. Chambers, J. I. Lunine, J. M. Petit, F. Robert, G. B. Valsecchi and K. E. Cyr, Meteoritics \& Planetary Science, 2000, 35, 1309-1320.

8. K. Zahnle, L. Schaefer and B. Fegley, Cold Spring Harbor Perspectives in Biology, 2010, 2.

9. J. A. Tyburczy, B. Frisch and T. J. Ahrens, Earth and Planetary Science Letters, 1986, 80, 201-207.

10. L. Remusat, S. Derenne and F. Robert, Geochimica et Cosmochimica Acta, 2005, 69, 4377-4386.

11. P. Schmitt-Kopplin, Z. Gabelica, R. D. Gougeon, A. Fekete, B. Kanawati, M. Harir, I. Gebefuegi, G. Eckel and N. Hertkorn, Proceedings of the National Academy of Sciences, 2010, 107, 2763-2768.

12. C. Koeberl, Elements, 2006, 2, 211-216. 
13. L. Schaefer and B. Fegley, Icarus, 2010, 208, 438-448.

14. W. B. Tonks and H. J. Melosh, Journal of Geophysical Research: Planets, 1993, 98, 5319-5333.

15. T. Kleine and J. F. Rudge, Elements, 2011, 7, 41-46.

16. J. Wade and B. J. Wood, Earth and Planetary Science Letters, 2005, 236, 7895.

17. J. W. Delano, Origins of life and evolution of the biosphere, 2001, 31, 311341.

18. D. Canil, Earth and Planetary Science Letters, 2002, 195, 75-90.

19. K. Nakamura and Y. Kato, Geochimica et Cosmochimica Acta, 2004, 68, 4595-4618.

20. N. H. Sleep and K. Zahnle, Journal of Geophysical Research: Planets, 2001, 106, 1373-1399.

21. J. C. G. Walker, Origins of life and evolution of the biosphere, 1985, 16, $117-$ 127.

22. J. Kasting and T. Ackerman, Science, 1986, 234, 1383-1385.

23. A. M. Hessler, D. R. Lowe, R. L. Jones and D. K. Bird, Nature, 2004, 428, 736-738.

24. J. F. Kasting, Precambrian Research, 2005, 137, 119-129.

25. M. J. NEWMAN and R. T. ROOD, Science, 1977, 198, 1035-1037.

26. I. Ribas, E. F. Guinan, M. Gudel and M. Audard, The Astrophysical Journal, 2005, 622, 680-694.

27. S. K. Atreya, Science, 2006, 316, 843-845.

28. B. Gans, S. Boye-Peronne, M. Broquier, M. Delsaut, S. Douin, C. E. Fellows, P. Halvick, J.-C. Loison, R. R. Lucchese and D. Gauyacq, Physical Chemistry Chemical Physics, 2011, 13, 8140-8152.

29. C. Romanzin, Y. Bénilan, A. Jolly and M. C. Gazeau, Advances in Space Research, 2008, 42, 2036-2044.

30. J. F. Kasting and J. C. G. Walker, Journal of Geophysical Research: Oceans, 1981, 86, 1147-1158.

31. F. Tian, J. F. Kasting and K. Zahnle, Earth and Planetary Science Letters, 2011, 308, 417-423.

32. F. Tian, O. B. Toon, A. A. Pavlov and H. De Sterck, Science, 2005, 308, 1014-1017. 
33. J. F. Kasting, Science, 1993, 259, 920-926.

34. E. O. Lippman, 1933.

35. A. Oparin, 1938.

36. J. B. S. Haldane, The Rationalist Annual, 1929, 148, 3-10.

37. K. A. Wilde, B. J. Zwolinski and R. B. Parlin, Science, 1953, 118.

38. H. C. Urey, Proceedings of the National Academy of Sciences, 1952, 38, 351363.

39. A. Lazcano and J. L. Bada, Origins of life and evolution of the biosphere : the journal of the International Society for the Study of the Origin of Life, 2003, 33, 235-242.

40. S. L. Miller, Science, 1953, 117, 528-529.

41. S. L. Miller and H. C. Urey, Science, 1959, 130, 245-251.

42. A. P. Johnson, H. J. Cleaves, J. P. Dworkin, D. P. Glavin, A. Lazcano and J. L. Bada, Science, 2008, 322, 404-404.

43. E. T. Parker, H. J. Cleaves, M. P. Callahan, J. P. Dworkin, D. P. Glavin, A. Lazcano and J. L. Bada, Origins of Life and Evolution of Biospheres, 2011, 41, 201-212.

44. M. L. Cable, S. M. Hörst, R. Hodyss, P. M. Beauchamp, M. A. Smith and P. A. Willis, Chemical Reviews, 2012, 112, 1882-1909.

45. B. N. Khare, C. Sagan, J. E. Zumberge, D. S. Sklarew and B. Nagy, Icarus, 1981, 48, 290-297.

46. M. G. Trainer, A. A. Pavlov, D. B. Curtis, C. P. McKay, D. Worsnop, A. E. Delia, D. W. Toohey, O. B. Toon and M. A. Tolbert, Astrobiology, 2004, 4, 409-419.

47. M. Trainer, Current Organic Chemistry, 2013, 17, 1710-1723.

48. B. Fleury, N. Carrasco, M. Millan, L. Vettier and C. Szopa, Earth and Planetary Science Letters, 2017, 479, 34-42.

49. C. N. Matthews and R. E. Moser, Nature, 1967, 215, 1230-1234.

50. F. Raulin, in L'environnement de la Terre primitive, eds. M. Gargaud, D. Despois and J. P. Parisot, Presses Universitaires de Bordeaux, Bordeaux, 2005, pp. 343-360.

51. B. Charnay, F. Forget, R. Wordsworth, J. Leconte, E. Millour, F. Codron and A. Spiga, Journal of Geophysical Research: Atmospheres, 2013, 118, 10,414410,431 . 
52. B. Fleury, N. Carrasco, E. Marcq, L. Vettier and A. Määttänen, The Astrophysical Journal, 2015, 807, L29.

53. K. Pérot, A. Hauchecorne, F. Montmessin, J.-L. Bertaux, L. Blanot, F. Dalaudier, D. Fussen and E. Kyrölä, Atmospheric Chemistry \& Physics, 2010, 10, 2723.

54. D. Lowe and A. R. MacKenzie, Journal of Atmospheric and Solar-Terrestrial Physics, 2008, 70, 13-40.

55. C. P. McKay, J. B. Pollack and R. Courtin, Science, 1991, 253, 1118.

56. L. Gavilan, L. Broch, N. Carrasco, B. Fleury and L. Vettier, The Astrophysical Journal Letters, 2017, 848, L5.

57. L. Gavilan, N. Carrasco, S. V. Hoffmann, N. Jones, C. and N. Mason, J., The Astrophysical Journal, 2018, 861, 110.

58. A. Mahjoub, N. Carrasco, P.-R. Dahoo, T. Gautier, C. Szopa and G. Cernogora, Icarus, 2012, 221, 670-677.

59. B. J. Mason, Contemporary Physics, 2002, 43, 1-11.

60. S. K. Atreya, Science, 2007, 316, 843-845.

61. S. Hörst, R. Yelle, A. Buch, N. Carrasco, G. Cernogora, O. Dutuit, E. Quirico, E. Sciamma-O'Brien, M. Smith, A. Somogyi, C. Szopa, R. Thissen and V. Vuitton, Astrobiology, 2012, 12, 809-817.

62. D. L. Matson, L. J. Spilker and J.-P. Lebreton, Space Science Reviews, 2002, 104, 1-58.

63. R. D. Lorenz, E. P. Turtle, J. W. Barnes, M. G. Trainer, D. S. Adams, Kenneth E. Hibbard, Colin Z. Sheldon, Kris Zacny, Patrick N. Peplowski, David J. Lawrence, Michael A. Ravine, Timothy G. McGee, Kristin S. Sotzen, Shannon M. MacKenzie, Jack W. Langelaan, Sven Schmitz, Lawrence S. Wolfarth and P. D. Bedini, Johns Hopkins APL Technical Digest, 2018, 34. 